\title{
STUDY OF THE BEHAVIOUR OF INHOMOGENEOUS HARMONIC WAVES IN THEIR DIFFRACTION FROM SIMPLE ROUGH SURFACES
}

\author{
R. BRIERS and O. LEROY \\ K.U.L. Campus Kortrijk, Interdisciplinair Research Center, B-8500 Kortrijk, Belgium
}

\begin{abstract}
A theoretical model is presented to investigate the diffraction of inhomogeneous waves from a traction-free solid-air boundary having simple periodic profile. It is shown how one can calculate the amplitudes, the angular positions and the exponential decays of the various diffracted orders. The numerical results for a brass-air boundary are compared with the experimental results obtained by Jungman et al.[1]. Our model accounts for the first reflection minimum near the fundamental surface resonance frequency, corresponding to Rayleigh wave generation. This minimum is clearly observed experimentally, but fails to show up in the theoretical results of homogeneous plane wave theory.

Résumé: Un modèle théorique est présenté pour investiguer la diffraction d'ondes planes inhomogènes par une surface périodique solide-air.On calcule les amplitudes, les positions angulaires et les décroissances exponentielles des ordres diffractés.Les résultats numériques pour le cas d'une interface cuivre-air sont comparés avec les résultats expérimentaux de Jungman et al.[1]. La théorie des ondes planes homogènes ne décrit pas le minimum du coefficient de réflection près de la fréquence fondamentale,correspondant à la génération de l'onde de Rayleigh,tandis que notre modèle prévoit un minimum prononcé.
\end{abstract}

\section{The general system of equations}

The geometry of the two-dimensional diffraction problem is sketched in Fig.1. The solid is assumed to be isotropic and homogeneous. The solid-air boundary is described by the equation

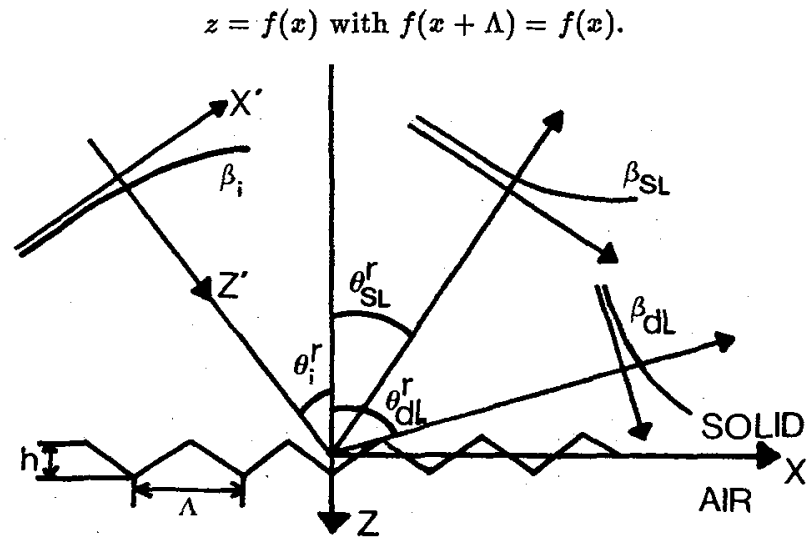

Figure 1: Geometrical configuration for the solid-air problem 
A longitudinal inhomogeneous wave with exponential decay $\beta_{i}$, phase velocity $\mathrm{v}$ and angle of incidence

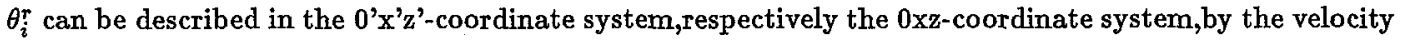
potential (the time dependence is suppressed further on)

$$
\varphi_{i}\left(x^{\prime}, z^{\prime}\right)=A \exp \left(\beta_{i} x^{\prime}\right) \exp \left(i \frac{\omega}{v} z^{\prime}\right)
$$

respectively

$$
\varphi_{i}(x, z)=A \exp \left[i\left(k_{i} x+K_{i} z\right)\right]
$$

with

$$
k_{i}=k_{d} \sin \left(\theta_{i}^{*}\right)=k_{d} \sin \left(\theta_{i}^{r}+i \theta_{i}^{c}\right), \quad K_{i}=k_{d} \cos \left(\theta_{i}^{*}\right), \quad k_{d}=\frac{\omega}{v_{d}},
$$

$\omega$ being the angular frequency of the radiation source and $v_{d}$ being the longitudinal velocity of the medium. The angle $\theta_{i}^{c}$ is related to the inhomogeneity $\beta_{i}$ and the wavenumber $k$ of the incident wave by

$$
\beta_{i}=-k \sinh \theta_{i}^{c}, \quad k=\frac{\omega}{v}=\frac{k_{d}}{\cosh \theta_{i}^{c}},
$$

from which we can deduce the dispersion relation

$$
k^{2}=k_{d}^{2}+\beta_{i}^{2} .
$$

The scattered dilatational $(m=d)$ and shear $(m=s)$ waves are represented by potentials $\varphi_{m}(x, z)$ which are solutions of the appropriate wave equations [2] and which must satisfy the continuity of stress

$$
\sum_{j=1}^{3} T_{i j}(\nabla \phi)_{j}=0, \quad i=1,3
$$

on the boundary $\phi(x, z)=0$ with $\phi(x, z)=f(x)-z$.

The potentials $\varphi_{m}(x, z)$ can be described by infinite series of inhomogeneous waves [3]

$$
\varphi_{m}(x, z)=\sum_{l=-\infty}^{\infty} M_{l} \exp \left[i\left(k_{l} x+K_{m l} z\right)\right]
$$

$M_{l}=A D_{l}$, or $M_{l}=A S_{l}$, where $D_{l}$ and $S_{l}$ are the unknown reflectioncoefficients of the 1-th order,

$$
k_{l}=\frac{\omega}{v_{m}} \sin \theta_{m l}^{*}=\frac{\omega}{v_{m}} \sin \left(\theta_{m l}^{r}+i \theta_{m l}^{c}\right), \quad K_{m l}=\frac{\omega}{v_{m}} \cos \theta_{m l}^{*},
$$

$v_{m}$ being the corresponding acoustic velocity of the medium. The same relations as in (5) and (6) can be written down for each scattered D-and S-wave of order 1.

The $\mathrm{x}$-components $k_{l}$ of the wave vectors are calculated from the complex diffraction equation

$$
k_{l}=k_{i}+l \frac{2 \Pi}{\Lambda}, l \in \mathscr{Z}
$$

Equating the real and imaginary part in both members, we obtain a nonlinear system from which the exponential decays $\beta_{m l}$ and the angular positions $\theta_{m l}^{r}$ of the diffracted D- and S-waves can be calculated

$$
\sqrt{k_{m}^{2}+\beta_{m l}^{2}} \sin \theta_{m l}^{r}=\sqrt{k_{d}^{2}+\beta_{i}^{2}} \sin \theta_{i}^{r}+l \frac{2 \Pi}{\Lambda}
$$




$$
\beta_{m l} \cos \theta_{m l}^{r}=\beta_{i} \cos \theta_{i}^{r}
$$

Using (2), (9) and (12) in the boundary conditions (7), we find a system of equations which are periodic functions of $x$ so that a sufficient condition for the solution is to require that the Fouriercoefficients are equal over $[0, \Lambda]$ :

$$
\begin{gathered}
\sum_{l=-\infty}^{\infty}\left[\left(k_{l}-k_{n}+2 \frac{v_{s}^{2}}{\omega^{2}} k_{n} K_{d l}^{2}\right] D_{l} I_{l n}^{d}+\sum_{l=-\infty}^{\infty} K_{s l}\left(2 k_{l} k_{n} \frac{v_{s}^{2}}{\omega^{2}}-1\right) S_{l} I_{l n}^{s}=\left[\left(k_{n}-k_{i}\right)-2 \frac{v_{s}^{2}}{\omega^{2}} k_{n} K_{i}^{2}\right] I_{o n}\right. \\
\sum_{l=-\infty}^{\infty} K_{d l}\left(1-2 k_{l} k_{n} \frac{v_{s}^{2}}{\omega^{2}}\right) D_{l} I_{l n}^{d}+\sum_{l=-\infty}^{\infty}\left[\left(k_{l}-k_{n}\right)+2 \frac{v_{s}^{2}}{\omega^{2}} k_{n} K_{s l}^{2}\right] S_{l} I_{l n}^{s}=K_{i}\left(2 k_{i} k_{n} \frac{v_{s}^{2}}{\omega^{2}}-1\right) I_{o n}
\end{gathered}
$$

with $n=\ldots-2,-1,0,+1,+2 \ldots$,

$$
I_{l n}^{m}=\int_{0}^{\Lambda} \exp \left[i\left(k_{l}-k_{n}\right)\right] \exp \left[i K_{m l} f(x)\right] \frac{1}{K_{m l}} d x
$$

and for $I_{o n}$ one has to require $k_{l}=k_{i}, \quad K_{m l}=K_{i}$.

\section{Numerical results and conclusions}

We applied our model for normal incidence to a brass sample with sawtooth corrugated boundary. The values of the physical and geometrical parameters are

$$
v_{d}=4700 \frac{m}{s}, v_{s}=2100 \frac{m}{s}, \Lambda=250 \mu m, h=66 \mu m .
$$

By truncating ( $-6 \leq l, n \leq 6)$ the infinite system of Eqs.(13)-(14),we calculated to a good accuracy the first 13 diffractioncoefficients $D_{l}, S_{l}$.

Fig.2 shows the numerical results for the zero order reflected longitudinal spectrum. We clearly see that plane wave theory $\left(\beta_{i}=0\right)$ doesn't predict the sharp dip around $8 \mathrm{MHz}$ that is observed experimentally [1]. Agreement between theory and experiment is obtained only by considering inhomogeneous waves. In the past, other authors $[1,4,5]$ modified the plane wave theory by introducing an appropriate gating function and finite beam analysis.

The reflectioncoefficient $D_{0}$ becomes zero for $\beta_{i}=0.1 \frac{1}{m m}$. By means of Eqs.(11)-(12), we obtain for the longitudinal reflected first order a diffraction angle $\theta_{d 1}^{r}=89.7^{\circ}$ and an exponential decay $\beta_{d 1}=$ $22.806 \frac{1}{\mathrm{~mm}}$, while for the transversal reflected first order we get the values $\theta_{s t}^{r}=89.3^{\circ}$ and $\beta_{s 1}=8.546 \frac{1}{\mathrm{~mm}}$. Remark that only for normal incident homogeneous plane waves, diffraction parallel with the surface can occur (see Eq.(12)). Using the appropriate dispersion relations (as in Eq.(6)) we find that each of the inhomogeneity parameters mentioned gives rise to a velocity of $1975 \frac{\mathrm{m}}{\mathrm{s}}$ along the periodic interface. For this reason we interprete those diffracted inhomogeneous waves as components of a first order generated Rayleigh wave $\left(v_{\text {Rayleigh }}=1980 \frac{\mathrm{m}}{\mathrm{s}}\right)$. That both components have a non-negligible amplitude can be seen on Figs. 4 and 5.

Another interesting phenomenon is shown on Fig.3. For normal incident homogeneous plane waves the zero order transversal reflectioncoefficient $S_{0}$ is always zero, while for inhomogeneous plane waves $S_{0}$ is not negligible at all and has a sharp extremum at the fundamental resonance frequency.

We also notice that all extrema in the spectra for inhomogeneous plane waves occur at one distinct frequency slightly shifted to the left compared with the frequency position of the extremum given by the homogeneous plane wave theory. 
This theory is extended for incident bounded beams described by means of series of inhomogeneous waves as well as for other configurations like liquid-solid and solid-liquid.
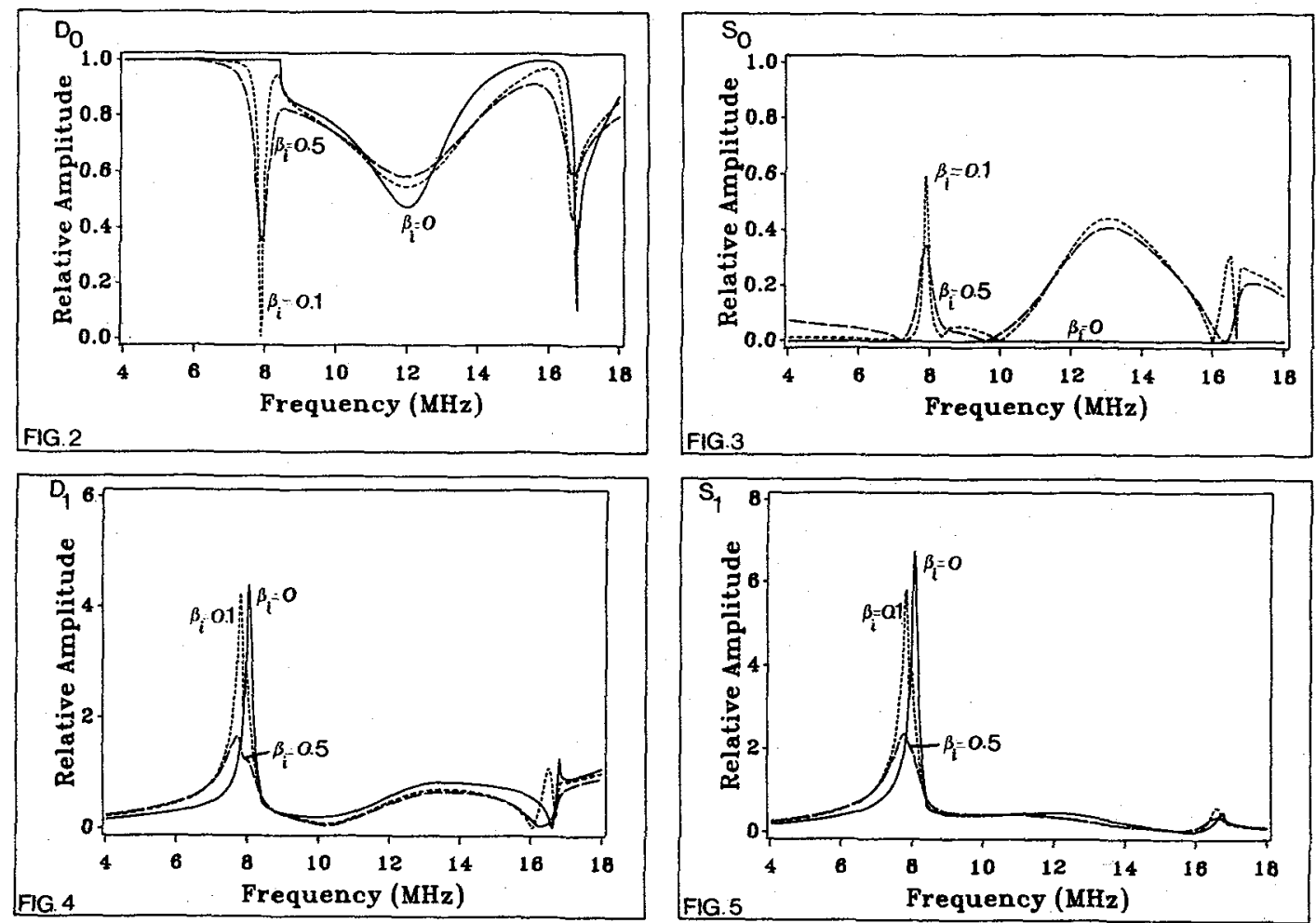

Figures 2 to 5 : zero and first order dilatational $\left(D_{0}, D_{1}\right)$ and shear $\left(S_{0}, S_{1}\right)$ reflected spectra for a brass-air interface $(h=66 \mu \mathrm{m}, \Lambda=250 \mu \mathrm{m})$ and for different values of the inhomogeneity parameter $\beta_{i}$

\section{References}

/1/Jungman A. et al.,J.Acoust.Soc.Am.74,1025-1032,1983.

/2/Brekhovskikh L.M.,Waves in Layered Media \$4,Academic Press,New York,1960.

/3/Claeys J.M. and Leroy O.,J.Appl.Pys.54,5657-5662,1983.

/4/Roberts R.,Achenbach J.D. et al.,Wave Motion 7,67-77,1985.

/5/Ko R.,Adler L. et al.,Quantitative Nondestructive Evaluation,1165-1172, Plenum Press,New York, 1984. 